\title{
Low complexity antenna diversity front-end - Use of code multiplexing -
}

\author{
Matthieu Gautier ${ }^{1,2}$ and Guillaume Villemaud ${ }^{1}$ \\ ${ }^{1}$ Université de Lyon, INRIA, INSA-Lyon, CITI - F-69621 - France \\ ${ }^{2}$ Orange Labs - 28, chemin du vieux chêne - 38243 Meylan - France
}

\begin{abstract}
In this paper, we address the architecture of an antenna diversity receiver and we aim to reduce the complexity of the analog front-end. To this end, an innovative architecture is introduced based on code multiplexing. This architecture uses the direct sequence spread spectrum technique in order to multiplex the different antennas contributions through a single demodulator. Simulation and measurement results show that, in a Gaussian case, the bit error rate does not increase so much with the multiplexing. The complexity evaluation shows that the proposed architecture significantly reduces the power consumption of the front-end.
\end{abstract}

\section{INTRODUCTION}

Introduced in 1984 by J.H. Winters [1], the antenna diversity techniques are an interesting solution for future communication systems. They are used to increase the capacity and performances (quality of service QoS, datarate, ... ) of wireless networks. Most studies on antenna diversity systems concern the digital processing part of the receiver physical layer. Thus, many digital algorithms [2][3] (beamforming, spacetime coding, spatial multiplexing, ... ) have been developed in order to increase capacity and performance. There are also studies on various types of antenna arrays [4] such as spatial diversity, pattern diversity, polarization diversity, ...

But the analog complexity issue concerning an antenna diversity receiver using digital antenna processing has been very little addressed [5]. In fact, the performance gain achieved by the diversity implies an increase of the digital complexity (algorithms implementation), but also an increase of the complexity and the consumption of the analog front-end because each additional antenna induces a complete additional analog branch [6].

Therefore this paper deals with the architecture of the analog front-end associated with these techniques. Introduced by authors in an international patent [7], we propose a novel architecture for antenna diversity receiver that reduces the complexity of the analog front-end. This architecture is based on code multiplexing.

In order to ensure the functionality of such a receiver, Bit Error Rate (BER) simulations have been performed and more realistic results have been measured by using an Agilent Technologies connected solution as presented in [8].

This paper consists of 5 parts. Following this introduction, Section II gives the motivation of studying this kind of architecture, then the new antenna diversity front-end is presented. In Section III, we evaluate the complexity and power consumption of the proposed architecture. Section IV details some (a)

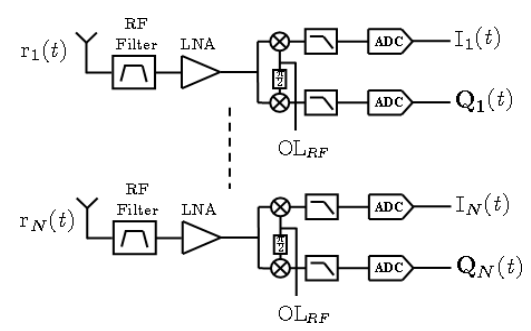

(b)

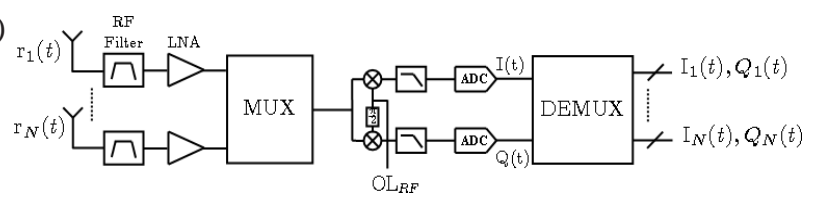

Fig. 1. Classical (a) and multiplexing (b) architectures of an antenna diversity receiver.

simulated and measured performances. Finally, conclusions are drawn and follow-ups are provided.

\section{The CODE MULTIPLEXING ARCHITECTURE}

\section{A. Motivation}

In order to have a performance improvement, a transceiver using antenna diversity [1] has to use several antennas for its transmitter and/or receiver. Fig. 1(a) shows the structure of the classical analog front-end of an antenna diversity receiver, it uses one dedicated analog chain for each of the $N$ antennas. Each of theses chains transforms the radiofrequency (RF) signal $r_{k}(t)$ in baseband signals in-phase $I_{k}(t)$ and inquadrature $Q_{k}(t)$ [9].

The receiver of the Fig. 1(a) uses a homodyne architecture [6]. Each chain is composed of an SAW RF filter (Surface Acoustic Wave filter) for the band selection, a LNA (Low Noise Amplifier) and an IQ demodulator that recovers the in-phase and in-quadrature baseband signals. The $I_{k}(t)$ and $Q_{k}(t)$ signals are then digitized by two analog to digital converters (ADC). This RF stack-up architecture is an obvious choice: having $N$ separate dedicated chains allows the demodulation of each branch with a significant quality (high Signal to Noise Ratio SNR at the ADC input). However, this choice enforces a high complexity of the analog front-end. The following work aims to reduce this complexity without decreasing the SNR quality after branches demodulation.

Therefor, this study explores the use of a single common frontend for the processing of signals received by the $N$ antennas. The use of one common analog chain underlies the idea of multiplexing the different branches on a single front-end. The 
signal of one branch must be separated from those of the other branches in order to facilitate the sharing of a single analog reception chain.

Fig. 1(b) illustrates the principle of the multiplexing applied to an antenna diversity receiver. We notice that the multiplexing operation (MUX) is implemented analogically, while the demultiplexing operation (DEMUX) is implemented digitally. We assess the application of the different multiplexing techniques to the antenna diversity receiver. The domains commonly used to share and diversify support are the temporal, frequency and code domains. The application of these multiplexing techniques to multi-antenna receiver gives the following analysis:

- The time domain: a theoretical structure has been proposed in [5] as a time multiplexing front-end architecture. The structure uses an RF switch which operates $N$ times quicker than the symbol period. The weakness of this approach is a SNR loss because only a fraction of the received power has been used by the demodulator. Moreover, implementation constraints of the RF switch are not considered in the study and should be a barrier for the development of this kind of architecture. Time multiplexing technique is interesting for RF signals that are received during time period that do not overlap.

- The frequency domain: in [5], Evans et al. prosose a MIMO (Multi-Input Multi-Output) receiver based on frequency multiplexing. Meanwhile, several weaknesses of this approach have been observed: the stringent filtering requirements and the need of additional RF local oscillators. Frequency multiplexing is required to receive RF signals whose frequency bands do not overlap.

- The code domain: the multi-antenna signals used in this study are received simultaneously on the same frequency band. By using orthogonal codes, these signals become very low correlated between them so that information from each antenna can be extracted. This method has just been recently approached in [10]. However, this theoretical paper assesses the problem of using nonorthogonal codes and does not assess the performances and the power consumption of the structure.

The code domain appears to be the most suited for an antenna diversity receiver. In order to achieve the spectrum overlapping, decorrelation can be done by the spread spectrum technique. The direct sequence spread spectrum (DSSS) technique is the basis of code division multiple access (CDMA) technology [11]. The spread spectrum allows a multiplexing which is neither time nor frequency, but a code multiplexing. The theoretical aspects of the proposed structure are described in the next part.

\section{B. System model of code multiplexing receiver}

In this section, the direct sequence spread spectrum is implemented in an antenna diversity receiver. The novel antenna diversity receiver using code multiplexing is depicted in Fig. 2 and consists in 2 parts: the analog multiplexing and the digital demultiplexing.

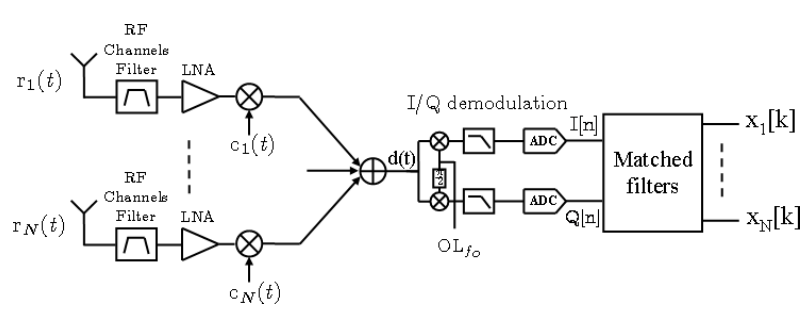

Fig. 2. Analog and digital front-end of the code multiplexing architecture.

Analog coding: The DSSS consists in allocating a spreading code to each branch, all these codes being orthogonal two by two. The received signal that carries information is multiplied by the code which is a pseudo-random sequence of $N$ binary entities having a rate $N$ times higher than the symbol. Thus, the resulting signal has variations that are $N$ times faster than the information signal, increasing $N$ times the bandwidth of the signal frequency spectrum.

To illustrate the code multiplexing, Fig. 3 shows the power spectrum of the signal before and after code multiplexing. We use a $N=4$ antennas system that receives an IEEE 802.11g [12] type signal having a $20 \mathrm{MHz}$ bandwidth and a $2412 \mathrm{MHz} \mathrm{RF}$ frequency. The frequency bandwidth after coding is $80 \mathrm{MHz}$. Indeed, the multiplex operation generates a $N=4$ bandwidth increase factor.
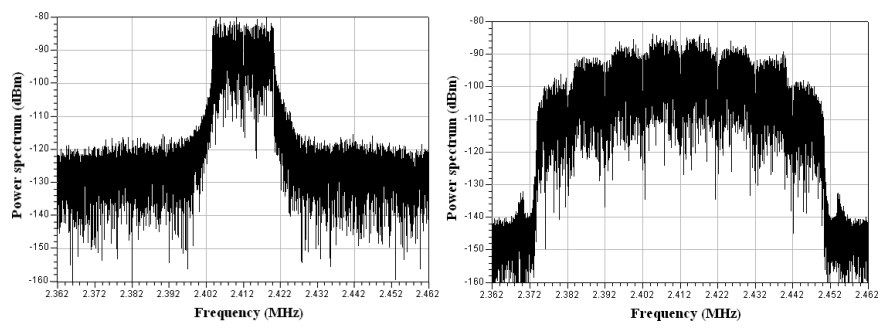

Fig. 3. Power spectral density before and after code multiplexing.

First, let us look at the $k^{t h}$ antenna contribution. The digital transmitted message is made of complex symbols $x_{k}[i]$ which depend on the digital modulation (QAM, OFDM, ...). These symbols are transmitted at the symbol rate $D_{s}=1 / T_{s}$ where $T_{s}$ is the symbol duration. The digital baseband signal (analog signal carrying digital information), with a rectangular pulse shaping, is written:

$x_{k}(t)=\sum_{i=-\infty}^{+\infty} x_{k}[i] p_{T_{s}}\left(t-i T_{s}\right)$ with $p_{T_{s}}=\left\{\begin{array}{l}1 \text { if } 0 \leq t<T_{s} \\ 0 \text { else }\end{array}\right.$

The transmitted signal is real and is defined by:

$$
s_{k}(t)=x_{k}(t) e^{j 2 \pi f_{0} t}+x_{k}^{*}(t) e^{-j 2 \pi f_{0} t},
$$

with $f_{0}$ the RF modulating frequency.

In an ideal transmission through a non-dispersive channel, the received signal is expressed by:

$$
r_{k}(t)=A_{k} s_{k}(t)+n_{k}^{R F}(t)
$$


with $A_{k}$ the path loss attenuation and $n_{k}^{R F}(t)$ the total RF noise at the input of the $k^{t h}$ antenna.

In order to perform the code multiplexing of all the branches, each received signal is spread using a code $c_{k}(t), k=1, \ldots N$. The pseudo-random sequences are $T_{s}$-periodic. By using a limited symbol duration code, repeated indefinitely, the system has an easier sequence generation as well as a better despreading synchronization. For a given branch $k$, a set of $N$ binary (complex binary) chips $\left\{c_{k}[n] n=0, \ldots N-1\right\}$ is used. This sequence is called "spreading code". The periodic code $c_{k}^{\text {per }}(t)$ is the periodic pseudo-random sequence:

$$
c_{k}^{\text {per }}(t)=\sum_{i=-\infty}^{+\infty} c_{k}(t) p_{T_{s}}\left(t-i T_{s}\right) .
$$

The non-periodic code is defined by:

$$
c_{k}(t)=\sum_{n=0}^{N-1} c_{k}[n] p_{T_{c}}\left(t-n T_{c}\right),
$$

where $T_{c}$ is the chip duration and $N=\frac{T_{s}}{T_{c}}$ is the code length. The use of codes with a $N$ length allows the reception of $N$ antennas.

The $k^{t h}$ antenna contribution after spreading $d_{k}(t)$ is expressed by:

$$
d_{k}(t)=A_{k} c_{k}^{p e r}(t) s_{k}(t)+c_{k}^{p e r}(t) n_{k}^{R F}(t) .
$$

$\mathrm{Bu}$ replacing $s_{k}(t)$ by (2), we get:

$d_{k}(t)=A_{k} c_{k}^{\text {per }}(t)\left\{x_{k}(t) e^{j 2 \pi f_{0} t}+x_{k}^{*}(t) e^{-j 2 \pi f_{0} t}\right\}+c_{k}^{p e r}(t) n_{k}^{R F}(t)$.

Once the coding operation ended for each antenna, the new signals intercorrelations depend only on the codes intercorrelations. So, signals can be clearly overlapped in time and frequency, as they are separable by their spreading sequence. The adding operation between the spread contributions can be done:

$$
d(t)=\sum_{k=1}^{N} d_{k}(t), \quad \text { avec } K \leq N .
$$

The spread spectrum of $d(t)$ can be seen in Fig. 3 .

After the code multiplexing step, the signal $d(t)$ is transposed to the baseband frequency by an IQ demodulator. $I(t)$ is the in-phase component and $Q(t)$ is the in-quadrature component. $L P[\bullet]$ refers to an ideal low-pass filter having a bandwidth of $B_{d} / 2\left(B_{d}\right.$ is bandwidth of $\left.d(t)\right) . I(t)$ and $Q(t)$ are expressed by:

$$
\begin{aligned}
I(t) & =L P\left[d(t) \cos \left(2 \pi f_{0} t\right)\right] \\
& =\sum_{k=1}^{N}\left\{\frac{A_{k}}{2} c_{k}^{p e r}(t)\left(x_{k}(t)+x_{k}^{*}(t)\right)+c_{k}^{p e r}(t) n_{k}^{R F}(t) \cos \left(2 \pi f_{0} t\right)\right\},
\end{aligned}
$$

$$
Q(t)=L P\left[d(t) \sin \left(2 \pi f_{0} t\right)\right]
$$$$
=\sum_{k=1}^{N}\left\{A_{k} \frac{j}{2} c_{k}^{p e r}(t)\left(x_{k}^{*}(t)-x_{k}(t)\right)+c_{k}^{p e r}(t) n_{k}^{R F}(t) \sin \left(2 \pi f_{0} t\right)\right\}
$$

After demodulation, the signal is defined by its complex envelope:

$$
\hat{x}(t)=I(t)+j Q(t)=\sum_{k=1}^{N}\left\{c_{k}^{\text {per }}(t) x_{k}(t)+c_{k}^{\text {per }}(t) n_{k}^{B B}(t)\right\},
$$

where $n_{k}^{R F}(t)=R e\left[n_{k}^{B B}(t) e^{j 2 \pi f_{0} t}\right]$.

Digital decoding: So far, the different steps will be implemented in the analog part of the receiver. The decoding step will be performed digitally.

The pseudo-random sequence modulating the symbols during the spreading step has to be known by the decoder in order to enable the reconstruction of symbols by successive correlations (despreading and integration on the symbol time) between the spreading signal and the same coding sequence:

$$
\begin{aligned}
\hat{x}_{l}[m]= & \frac{1}{T_{s}} \int_{m T_{s}}^{(m+1) T_{s}} \hat{x}(t) c_{l}^{*}(t) d t, \quad m T_{s} \leq t<(m+1) T_{s} \\
= & \frac{1}{T_{s}} \sum_{k=1}^{N} \int_{m T_{s}}^{(m+1) T_{s}} c_{k}(t) c_{l}^{*}(t)\left\{A_{k} \sum_{i=-\infty}^{+\infty}\left(x_{k}[i] p_{T_{s}}\left(t-i T_{s}\right)\right)\right. \\
& \left.+n_{k}^{B B}(t)\right\} d t, \\
= & \frac{1}{T_{s}} \sum_{k=1}^{N}\left\{A_{k} x_{k}[m]+n_{k}^{B B}(t)\right\} \int_{0}^{T_{s}} c_{k}(t) c_{l}^{*}(t) d t .
\end{aligned}
$$

Intercorrelation properties influence the performances (detection and synchronization) of a spread spectrum system that operates by correlation between signals and codes. Intercorrelation functions of the codes $\gamma_{k, l}(\tau)$ are defined by:

$$
\gamma_{k, l}(\tau)=\frac{1}{T_{s}} \int_{0}^{T_{s}} c_{k}(t-\tau) c_{l}^{*}(t) d t
$$

By replacing (18) in (17), we obtain:

$$
\hat{x}_{l}[m]=\sum_{k=1}^{N}\left\{A_{k} x_{k}[m]+n_{k}^{B B}(t)\right\} \gamma_{k, l}(0) .
$$

If the codes are orthogonals, we have:

$$
\gamma_{k, l}(0)=\delta[k-l]
$$

As a final result, we get:

$$
\begin{aligned}
\hat{x}_{l}[m] & =\sum_{k=1}^{N}\left\{A_{k} x_{k}[m]+n_{k}^{B B}(t)\right\} \delta[k-l], \\
& =A_{l} x_{l}[m]+n_{l}^{B B}(t) .
\end{aligned}
$$

The digital baseband symbols $\left\{x_{l}[m], l=1 \ldots K, m \in \mathbb{Z}\right\}$ received on each antenna are recovered.

The proposed architecture works for any multi-antenna schemes: for every antenna array and every digital algorithm. However, a limitation of the concept of using orthogonal spreading codes is that only an even number $(2,4,8, \ldots)$ of antennas can be received. The synchronization between coding and decoding is not such an important issue as during an UMTS transmission. Indeed, the propagation delay of the 
spread signal through the circuit path is well-predicted through accurate circuit analysis and simulation. Therefore, attaining synchronization between the spreading and despreading codes is a trivial matter. For this reason, time delay between the spreading and despreading codes was neglected in this study.

\section{COMPlEXity STUDY}

We will now assess the complexity gain of the proposed structure (Fig. 2) compared to the classical architecture shown in Fig. 1(a). First, a comparison study, in terms of number of components, has been realized. Once this step finished, another comparison study involving the power consumption of the analog front-end is done.

\section{A. Complexity evaluation}

Fig. 2 shows a complete structure of the analog code multiplexing receiver. Each branch is made of an antenna, an RF filter and a LNA. The LNA output signals are mixed with the codes before being added. The multiplex RF signal is then transposed to baseband frequency by an IQ demodulator.

Table I reveals the number of components required for each structure and some constraints associated with these components. $N$ is the number of branches, $B_{c}$ is the channel bandwidth, $f_{0}$ is the RF standard center frequency and $B W$ stands for bandwidth.

\begin{tabular}{c|c|c|c|c|}
\cline { 2 - 5 } \multicolumn{1}{c|}{} & \multicolumn{2}{c|}{ Classical structure } & \multicolumn{2}{c|}{ Code multiplexing structure } \\
\cline { 2 - 5 } & Number & Specification & Number & Specification \\
\hline \hline Antenna & $N$ & $B W=B_{c}$ & $N$ & $B W=B_{c}$ \\
\hline Filter & $N$ & $B W=B_{c}$ & $N$ & $B W=B_{c}$ \\
\hline LNA & $N$ & $B W=B_{c}$ & $N$ & $B W=B_{c}$ \\
\hline Mixer & $2 N$ & $f_{0}$ & $N+2$ & $f_{0}$ \\
\hline Adder & 0 & & 1 & $N$ to 1 \\
\hline ADC & $2 N$ & $B W=B_{c}$ & 2 & $B W=N B_{c}$ \\
\hline
\end{tabular}

TABLE I

NUMBER OF COMPONENTS FOR THE FRONT-END USING A CLASSICAL AND A CODE MULTIPLEXING ARCHITECTURE.

Regardless of technological constraints, the major difference between the two structures is the number of mixers and ADC. The proposed structure reduces by a $N$ factor the number of ADC compared to the classical structure. In order to take into account the technological constraints, we propose a power consumption evaluation in the next section.

The digital complexity of the matched filters ( $N$ filters using $N$ coefficients) is neglected in this analysis. The reason is that the additional complexity of this processing is not important compared to the initial complexity of algorithms needed to perform the antenna diversity processing.

The code synthesis is an important part of the structure which is not presented in this paper. A digital generation of the code has been proposed as a solution, its complexity is also neglected in this analysis.

\section{B. Power consumption}

To evaluate the power consumption, we consider a standard that can use multi-antenna receiver: IEEE 802.11g having a channel bandwidth of $B=20 \mathrm{MHz}$.
Among the components of Table I, the antennas, the filters and the adder are passive components while the LNA have exactly the same characteristics for both classical and code multiplexing architecture. Therefore, this study takes into account the power consumption $P_{W}$ only for the mixers and the ADC:

$$
P_{W}=N_{\text {Mixer }} P_{\text {Mixer }}+N_{A D C} P_{A D C},
$$

where $N_{\text {Mixer }}$ and $N_{A D C}$ are the number of the mixers and of the ADC given in Table I. $P_{\text {Mixer }}$ and $P_{A D C}$ are the consumption of one mixer and ADC respectively.

As a mixer, we propose to use the mixer MAX 2682 from MAXIM for the 2 structures. This component has a power consumption $P_{\text {Mixer }}=45 \mathrm{~mW}$.

To determine $P_{A D C}$, we use the figure of merit given by [13]:

$$
F o M_{A D C}=\frac{2^{E N O B} \times 2 \times f_{s}}{P_{A D C}},
$$

with $E N O B$ the effective number of bits (we choose $E N O B=10$ bits [6]) and $f_{s}$ the sample rate $\left(f_{s}=4 B_{s}\right.$ for IEEE $802.11 \mathrm{~g}$ standard with $B_{s}$ the sampling bandwidth). The value given by [13] for 2007 is $F o M_{A D C}=1200 \mathrm{GHz} / \mathrm{W}$. $B_{s}$ is equal to $B$ for the classical architecture and is equal to $N B$ for the code multiplexing architecture.

Table II gives the power consumption $P_{W}$ for different numbers $N$ of antennas.

\begin{tabular}{|c|c|c|}
\hline$N$ & Classical structure & Code multiplexing structure \\
\hline \hline 1 & $0.158 \mathrm{~W}$ & $0.203 \mathrm{~W}$ \\
\hline 2 & $0.317 \mathrm{~W}$ & $0.317 \mathrm{~W}$ \\
\hline 4 & $0.633 \mathrm{~W}$ & $0.543 \mathrm{~W}$ \\
\hline 8 & $1.266 \mathrm{~W}$ & $0.996 \mathrm{~W}$ \\
\hline
\end{tabular}

TABLE II

POWER CONSUMPTION FOR IEEE 802.11G RECEPTION.

Results show that the proposed structure reduces significantly the power consumption of an antenna diversity front-end. This structure outperforms the classical structure for a number of antennas above 2 . A consumption reduction of $25 \%$ is reached for $N=8$ antennas.

\section{Simulated AND EXPERIMENTAL VALIDATION}

The functionality of such a receiver is validated in this part by using several BER simulations and measurements. First, two complete IEEE 802.11g [12] transmission systems have been modeled using the Advanced Design System (ADS) software [14]: one using the classical homodyne front-end, the other using the code multiplexing front-end. Then a connected solution [8] has been realized for both solutions in order to obtain realistic measures.

\section{A. System description}

The implemented system is described in Fig 2. Each antenna receives an IEEE $802.11 \mathrm{~g}$ type signal. The channel bandwidth is $20 \mathrm{MHz}$, the RF frequency is $2412 \mathrm{MHz}$ and the datarate is $6 \mathrm{Mbit} / \mathrm{s}$. The signals $r_{k}(t)$ are multiplied by the periodical spreading codes $c_{k}(t)$. The sum of $N$ encoded signals 


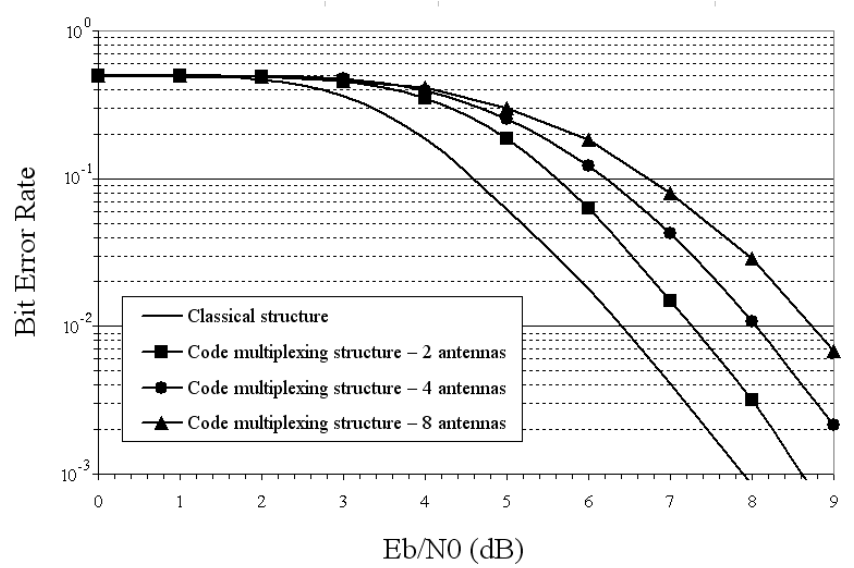

Fig. 4. BER versus $\frac{E_{b}}{N_{0}}$ for different number of antennas.

is then performed in order to generate the radio-frequency multiplex signal $d(t)$. This signal is then transformed by an IQ demodulator. After the sampling step, we apply matched filters consisting in a digital filter (impulse response $c_{k}^{*}[n]$ ) followed by a subsampling operation.

We suppose that the received signal provided by an antenna is independent of those received from the other antennas. We also consider a perfect code synchronization. We choose WalshHadamard codes [15] as those used by the UMTS standard. The signal $r_{1}(t)$ from the first antenna is not spread because its associated code $c_{1}(t)$ is only composed of ' 1 '. Thus, the performances are given only for the second antenna which is coded by a non-unitary code $c_{2}(t)$.

\section{B. Simulations results}

Simulated performances of the code multiplexing architecture are compared to the performances of a classical homodyne structure. The BER evolutions as a function of $\frac{E_{b}}{N_{0}}$ are shown in Fig. 4. The figure shows the influence of the number of multiplexed antennas on the BER evolution. A $N=2, N=4$ and $N=8$ antennas receiver are tested. We consider only the transmission quality of the second antenna, but the results are equal for each the antenna.

Simulations results show that, in an ideal no imperfections case (no multipath channel, no RF impairments), the structure using code multiplexing decreases the performances by less than $1 \mathrm{~dB}$ at a $B E R=10^{-2}$ for a $N=2$ antennas receiver. The $\frac{E_{b}}{N_{0}}$ degradation increases with the number of antennas, it reaches $2.3 \mathrm{~dB}$ for a $N=8$ antennas system. This degradation is due to digital aliasing during the subsampling step after filtering. Future work is to reduce this aliasing effect.

\section{Experimental results}

For the experimental validation [8], we use a radio platform described in Fig. 5. This platform is made of high technology equipments developed by Agilent Technologies [14]: the ADS software and measurement hardware which are two arbitrary waveform generators (ESG 4438C) and a vector spectrum analyzer (VSA89641) having two RF inputs. The arbitrary

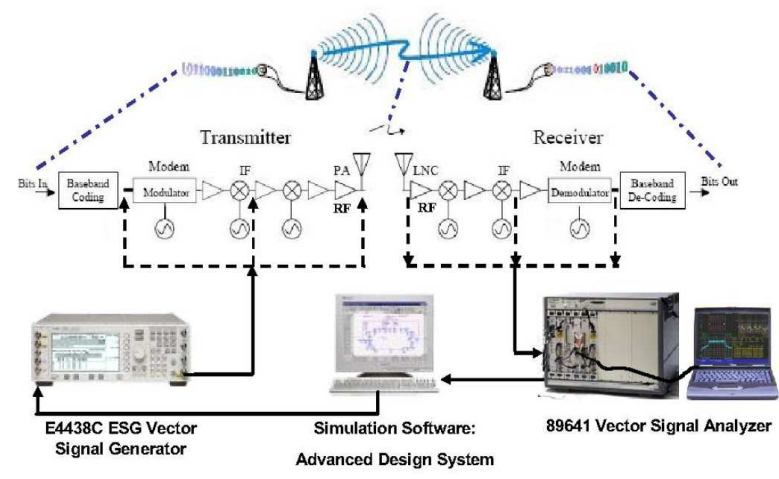

Fig. 5. The platform structure for a $1 \mathrm{x} 1$ transmission.

waveform generator is able to generate any complex signal which is then possible to analyze after propagation with the vector spectrum analyzer. The vectorial analysis software can demodulate this signal in order to accurately estimate the transmission system quality.

With this connected solution, a software/hardware interaction allows us to test and conceive very complex and realistic systems. We can therefore estimate the impact of the different noise sources of the RF front-end (phase noise, distortion, IQ imbalance, ...) and also the impact of the propagation environment (additive white Gaussian noise - AWGN , multipath, fading, ... ).

Using the two RF inputs of the VSA allows us to analyze and evaluate the performance of an antenna diversity system using two antennas. So, the experimental measurements are performed for both the classical and the code multiplexing architecture using $N=2$ antennas.

BER measurements are realized for an AWGN channel and for different SNR of the antennas inputs. The measures are given for both the code multiplexing architecture and the classical homodyne structure and are compared with the performances obtained by simulation in Fig 4. The BER evolutions of the second antenna are shown in Fig. 6.

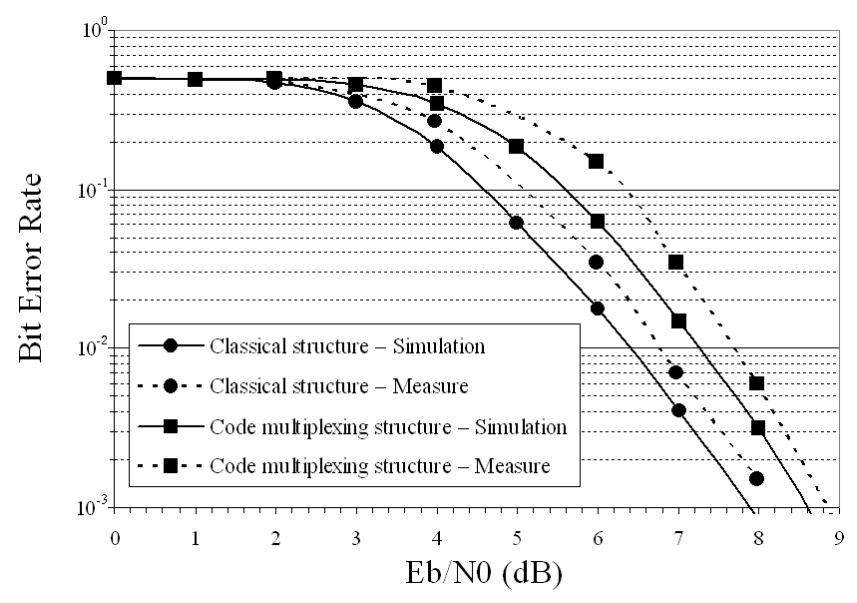

Fig. 6. Simulated and measured BER versus $\frac{E_{b}}{N_{0}}$ for a 2 antennas receiver. 
Compared to the simulated results, the measured results are somewhat degraded, the difference is due to the channel used for the measurement which may not exactly be an AWGN one. The $\frac{E_{b}}{N_{0}}$ gap between the classical and code multiplexing structures is almost the same during the measurements as that obtained during the simulations. It turns around $1 \mathrm{~dB}$ for a $B E R=10^{-2}$.

\section{Conclusions}

In this paper, we present a novel architecture for an antenna diversity receiver. The proposed structure uses orthogonal codes to multiplex the different branches through a single IQ demodulator. The main objective was to reduce the complexity of the analog front-end.

The first points revealed in this study are the evaluation the complexity and power consumption gain of the architecture. The system reduces the number of ADC by using only two ADC instead of the $2 N$ used by the classic receiver. Meanwhile, specifications of the ADC in terms of bandwidth are much more stringent.

Then, we demonstrate the feasibility of such a structure. The implementation of analog coding and digital decoding has been validated by BER simulations and measurements.

The follow-ups of this work are to accurately define the specifications of the analog components and to study the influence of their defaults (non-linearity, IQ imbalance) on the demodulation quality. An extension to multi-channel receiver has to be studied as well as its resulting complexityperformance trade-off.

A patent is pending on the proposed architecture [7].

\section{ACKNOWLEDGMENT}

The authors would like to thank Orange Labs which supporte this study.

\section{REFERENCES}

[1] J.H. Winters, "Optimum Combining in Digital Mobile Radio with Cochannel Interference," IEEE Journal on Selected Areas in Communications, vol. 2, no. 4, pp. 528-539, July 1984.

[2] S.M. Alamouti, "A Simple Transmit Diversity Technique for Wireless Communications," IEEE Journal on Selected Areas in Communications, vol. 16, no. 8, pp. 1451-1458, October 1998.

[3] I. E. Telatar, "Capacity of Multi-Antenna Gaussian Channels," AT\&T Bell Labs., Murray Hill, NJ, Intern. Tech. Memo, June 1995.

[4] C.B. Dietrich, K. Dietze, J.R. Nealy, and W.L. Stutzman, "Spatial, Polarization and Pattern Diversity for Wireless Handheld Terminals," IEEE Transactions on Antennas and Propagation, vol. 49, no. 9, pp. 1271-1281, September 2001.

[5] T. Kaiser, A. Bourdoux, H. Boche, J. Rodriguez Fonollosa, J. Bach Andersen, and W. Utschick, Smart Antennas: State of the Art, Eurasip Book Series on Signal Processing \& Communications, 2005.

[6] H. Tsurumi and Y. Suzuki, "Broadband RF stage architecture for software-defined radio inhandheld terminal applications," IEEE Communications Magazine, vol. 37, no. 2, pp. 90-95, February 1999.

[7] M. Gautier and G. Villemaud, Diversity receiver: analog complexity reduction by using mutiplexing technique, International patent pending (INPI number: 0759667), applicant: Orange Lab, December 2007.

[8] P.F. Morlat, X. Gallon, and G. Villemaud, "Measured Performances of a SIMO Multi-Standard Receiver," European Conference on Antennas and Propagation EUCAP07, Edinburgh, November 2007.

[9] J.G. Proakis, Digital communications, Mc Graw Hill internationnal editions, 3rd edition, 1995.
[10] F. Tzeng, A. Jahanian, and P. Heydari, "A Universal Code-Modulated Path-Sharing Multi-Antenna Receiver," IEEE Wireless Communication and Networking Conference (WCNC08), March 2008.

[11] R. Kohno, R. Meidan, and L.B. Milstein, "Spread spectrum access methods for wireless communications," IEEE Communications Magazine, vol. 33, no. 1, pp. 58-67, Janvier 1995.

[12] "Wireless MAN Medium Access Control and Physical Specification," IEEE Std 802.11, 1999.

[13] International Technology Roadmap for Semiconductors 2007 Edition, "System drivers".

[14] www.agilent.com.

[15] H. Schulze and C. Lueders, Theory and Applications of OFDM and CDMA: Wideband Wireless Communications, Wiley, 2005. 